\title{
Modeling of DSTATCOM Devices to Improve Dynamic Voltage Stability in a Microgrid with High Penetration of Motor Loads
}

\author{
Modelado de Dispositivos DSTATCOM para Mejorar la Estabilidad \\ Dinámica de Tensión en una Microrred con Alta Penetración de Cargas \\ Motóricas
}

\author{
L.A. Paredes ${ }^{1}$ \\ B.R. Serrano ${ }^{1}$ \\ M.G. Molina ${ }^{1}$ \\ ${ }^{1}$ Instituto de Energía Eléctrica, Universidad Nacional de San Juan - CONICET, San Juan, Argentina \\ E-mail:lparedes@iee.unsj.edu.ar; bserrano@iee-unsjonicet.org; mmolina@iee-unsjconicet.org
}

\section{Abstract}

This paper proposes and validates models of FACTS technology devices of the Synchronous Static Compensator for Distribution (DSTATCOM) type. In the first instance, a simplified DSTATCOM modeling performed through a controlled current source is presented. Subsequently, a DSTATCOM modeled through control strategies is exposed considering a PWM (Pulse-Width Modulation) converter. The proposed models of DSTATCOM devices are validated in a CIGRÉ Electrical Microgrid (MG) test system. The presence of induction motor type loads in the MG requires a considerable demand for reactive power, this causes inconveniences to maintain the Dynamic Voltage Stability (DVE). This situation is aggravated by the occurrence of failures that trigger an isolated operation of the MG. To solve these problems, a DSTATCOM device will be used. The optimal connection and location of DSTATCOM in the MG is done through an exhaustive search algorithm, considering the premise of the best performance in terms of DVE made through dynamic simulations in PowerFactory DIgSILENT. Comparatively, the results obtained for the two models performed are presented, showing the improvement of the DVE in the isolated operation of the MG with both models. Depending on the detail and scope of the studies in the MG system, you can choose between the developed DSTATCOM models. All details of models, control diagrams and data of DSTATCOM devices are provided in this document.

\section{Resumen}

A través de este artículo se propone y valida modelos de dispositivos de tecnología FACTS de tipo Compensador Estático Sincrónico para Distribución (DSTATCOM). En primera instancia, se presenta el modelado de un DSTATCOM simplificado realizado a través de una fuente de corriente controlada. Posteriormente, se expone un DSTATCOM modelado a través de estrategias de control considerando un conversor PWM (Pulse-Width Modulation - Modulación por ancho de pulsos). Los modelos propuestos de dispositivos DSTATCOM se validan en un sistema de prueba de Microrred Eléctrica (MRE) de la CIGRÉ. La presencia de cargas de tipo motor de inducción en la MRE requiere una considerable demanda de potencia reactiva, esto ocasiona inconvenientes para mantener la Estabilidad Dinámica de Tensión (EDT). Ésta situación se agrava ante la ocurrencia de fallas que desencadenen una operación aislada de la MRE. Para solucionar estos inconvenientes se hará uso de un dispositivo DSTATCOM. La conexión y ubicación óptima del DSTATCOM en la MRE se realiza a través de un algoritmo de búsqueda exhaustiva, considerando la premisa del mejor rendimiento en términos de EDT realizado a través de simulaciones dinámicas en PowerFactory DIgSILENT. Comparativamente se presentan los resultados obtenidos para los dos modelos realizados, demostrándose la mejora de la EDT en la operación aislada de la MRE con ambos modelos. Dependiendo del detalle y alcance de los estudios en el sistema de MRE, se puede elegir entre los modelos DSTATCOM desarrollados. Todos los detalles de modelos, esquemas de control y datos de los dispositivos DSTATCOM son proporcionados en este documento.

Palabras clave-- DSTATCOM, FACTS, Modelado, Estabilidad Dinámica de Tensión, Microrred, Motor de Inducción.
Index terms- DSTATCOM, FACTS, Modeling, Dynamic Voltage Stability, Microgrid, Induction Motor. 


\section{INTRODUCCIÓN}

El desarrollo sostenible de una sociedad depende en gran medida del sector energético de electricidad y representa un objetivo estratégico en cada país para consolidar un futuro seguro y sólido [1]. Los recursos energéticos tradicionales (petróleo y agua) para la generación de electricidad se están agotando, el nivel de contaminación del medio ambiente se vuelve cada más alarmarte por la elevada emisión de $\mathrm{CO}_{2}$ a la atmosfera, lo que ha desencadenado en las labores de investigación y desarrollo para aprovechar nuevas fuentes de generación de electricidad que sean amigables con el medio ambiente, así como también, nuevas formas de transportar y consumir energía de manera más eficiente y minimizando las pérdidas.

Los clásicos sistemas de distribución suministran energía eléctrica de manera unidireccional desde la red de transporte hacia los consumidores. La red de distribución se considera una red pasiva, con una configuración topológica radial. La red de distribución activa por su lado, tiene una configuración topológica en anillo o lazo cerrado, permitiendo que los flujos de potencia sean transportados de manera multidireccional, esto supeditado a la presencia de sistemas de Generación Distribuida (solar fotovoltaica, eólica, hidráulica, geotérmica, etc.), con lo cual se puede gestionar energéticamente el suministro eléctrico a los consumidores [1].

Los sistemas de Generación Distribuida (GD) y Microrredes Eléctricas (MREs), en las dos últimas décadas han recibido una connotada atención, posicionamiento, desarrollo e investigación en los mercados eléctricos. Esto impulsado por cuestiones ambientales, la necesidad de acceso a la energía eléctrica en comunidades remotas y la promesa de una mayor confiabilidad, seguridad, calidad y resiliencia de los sistemas eléctricos [2], [3]. Específicamente, las MREs se consideran un vínculo crítico o un punto de inflexión en la evolución de Sistemas de Suministro de Energía Eléctrica (SSEE) integrados verticalmente hacia las redes inteligentes descentralizadas (smart grids, en inglés) al facilitar la integración de la GD basados en Energías Renovables No Convencionales (ERNCs) [4], [5].

Un problema a tener en consideración en las MREs con fuentes de ERNCs debido a la GD son las constantes fluctuaciones de tensión. Estas fluctuaciones también pueden originarse por las inyecciones temporarias de potencia cuando una MRE se encuentra conectada a una red de distribución principal que suministra energía a la MRE. Adicionalmente, se debe considerar que las fluctuaciones de potencia son debidas a los sistemas de GD de tipo Solar Fotovoltaica (SFV), Generación Eólica (GE) y Sistemas de Almacenamiento de Energía (SAE), que conforman el sistema de MRE [6].

En concordancia a lo manifestado en [7], se cita que pueden ocurrir cambios significativos en los perfiles de tensión y frecuencia en una MRE, cuando ocurren transiciones del modo operativo red-MRE hacia el modo operativo de MRE aislada. En el modo operativo redMRE, la tensión y la frecuencia son impuestas principalmente por la red principal, limitando el rol de la MRE para realizar servicios auxiliares de regulación de estas dos variables eléctricas. Según lo establecido en el estándar IEEE 1547 [8], se permite la operación en isla de la MREs. En este contexto, la tensión y la frecuencia del sistema ya no son admitidos o referenciados por la red principal, lo que conlleva a que los diferentes sistemas de GD que conforman la MRE deban mantener estas variables en rangos aceptables garantizando la estabilidad de la MRE.

Para los sistemas de GD que conforman una MRE la toma de decisiones en relación a las inyecciones de potencia dependen en gran medida de la disponibilidad de los recursos energéticos primarios. En lo que respecta a las acciones de control que deben gestionar la operatividad de los componentes de GD en la actualidad un área relativamente inexplorada, ahondándose la problemática cuando se analiza la estabilidad ya sea de tensión o frecuencia de las MREs en sus modos operativos: asilados o de tipo interconectado red-MRE.

Típicamente, las cargas motóricas representan entre el $60-70 \%$ del consumo energético de un sistema de suministro de electricidad [9]. Particularmente los Motores de Inducción (MI) son ampliamente utilizados en los sectores industrial y residencial por su estructura simple, operación confiable y bajo costo [10]. Por lo tanto, considerar este tipo de cargas dinámicas en los sistemas de MREs se torna fundamental, con la premisa de que las mismas requieren una considerable demanda de potencia reactiva para su correcto funcionamiento.

Problemas asociados a la estabilidad de tensión en MREs y particularmente teniendo en consideración la presencia de cargas dinámicas de tipo MI se han abordado en algunas investigaciones [10], [11],

En la investigación [10] se presenta un modelo matemático que ejemplifica el proceso de arranque de un MI en una MRE y su efecto en la estabilidad transitoria. En [11], se aborda el problema de estabilidad dinámica de tensión considerando el impacto de la carga de tipo MI en una MRE en isla durante la ocurrencia de falla. Se analiza una estrategia de control enfocada en el soporte de potencia reactiva para un SFV en el entorno de Matlab/Simulink. En lo que respecta a la implantación de un dispositivo DSTATCOM en un sistema de distribución, en el trabajo [12], se analiza a través de simulaciones electromagnéticas el comportamiento de un DSTATCOM, como controlador de tensión y factor de potencia en un sistema de distribución que cuenta con cargas de tipo MI y estáticas.

Con los antecedentes mostrados, en el presente artículo se expone a través de un sistema de prueba de microrred, como incide la presencia de cargas motóricas 
en las dificultades para mantener el control y estabilidad dinámica de tensión, cuando ocurren contingencias que desencadena la operación asilada del sistema. Los modelos desarrollados y en función de los resultados obtenidos de las simulaciones dinámicas, se muestra comparativamente que, ya sea con los dispositivos DSTATCOM de tipo modelo simplificado y detallado, hay mejoras en la estabilidad dinámica de tensión de la microrred eléctrica en su modo operativo aislado.

El resto del presente artículo está organizado de la siguiente manera: en la sección dos, se presenta una visión panorámica de microrredes eléctricas y generación distribuida. Posteriormente, en la sección tres, se aborda la problemática asociada a la estabilidad de tensión en MREs. En la sección cuatro, se exhibe la tecnología FACTS y el modelado de ambos dispositivos DSTATCOM. Consecuentemente en la sección cinco, se presentan los casos de estudio, simulaciones y resultados comparativos obtenidos. Finalmente, en la sección seis, se exhiben las conclusiones y trabajos futuros derivativos del artículo.

\section{MICRORREDES ELÉCTRICAS Y CARGAS DINÁMICAS}

El DOE (Department Of Energy, departamento de energía de los Estados Unidos, por sus siglas en inglés) define a una microrred eléctrica así: "Es un conjunto de cargas interconectadas y recursos de energía distribuida que trabajan dentro de límites eléctricos definidos, que actúa como una única entidad controlable con respecto a la red y que se conecta o desconecta de dicha red para permitirle operar en modo conectado a la red o en modo de isla (autónomo)" [13]. Por otro lado, la CIGRÉ (Consejo Internacional de Grandes Redes Eléctricas, por sus siglas en francés), define a la MRE como: "son sistemas de distribución de electricidad que contienen cargas y recursos de energía distribuidos en forma de generación distribuida, dispositivos de almacenamiento y cargas controlables, que pueden operarse de forma controlada y coordinada, ya sea mientras están conectados a la red principal de energía o en modo de isla". [14].

En la actualidad, la tecnología de GD ha ido tomando un considerable posicionamiento, esto debido al aumento constante de diferentes fuentes de energías renovables, que no necesariamente son grandes parques de generación, sino más bien, están cercanas eléctricamente hablando a las cargas, lo que permite aprovechar de mejor manera el tendido eléctrico de las redes de distribución en media y baja tensión. Entre las principales ventajas que presenta este tipo de generación están: mejores índices de interrupción del suministro eléctrico, reducción de costos y precios de energía, mitigar la congestión del sistema de transmisión, reducción de emisión de gases al medioambiente y fortalecer la seguridad energética [14]. Es preciso indicar que la GD abarca diferentes tecnologías y sistemas, cuyas fuentes principales son recursos primarios tanto renovables como no renovables entre los que se incluyen recursos fósiles.

Son sistemas de GD aquellos cuya potencia instalada abarca entre las unidades de kilovatios hasta las decenas de megavatios, los cuales pueden estar instalados para su operación directamente a la red de distribución o conectados en el punto de suministro del usuario [15]. En función de la potencia instalada y capacidad de producción, los sistemas de GD se dividen en cuatro categorías: micro $[1 \mathrm{~W}-50 \mathrm{~kW}]$, pequeño [5kW-5MW], mediano [5-50MW] y grande [mayor de 50 MW] [16].

\subsection{Carga Dinámica de tipo Motor de Inducción}

La carga de un motor de inducción debe modelarse de tal manera que resulte sensible a las variaciones dinámicas en la tensión y frecuencia. En la literatura se recomienda el modelo de carga compuesto, como el más usual para simular dinámicamente esta carga en un sistema eléctrico. Este modelo consiste en dos partes, un motor de inducción, generalmente que obedezca a un modelo de tercer orden, en conjunto con una conexión paralela que represente la carga estática. El modelo de este motor es mostrado en la Fig. 1. El comportamiento dinámico de un MI de tercer orden obedece a las ecuaciones mostradas en (1) y cuya descripción detallada de parámetros y modelos son mostrados en [17].

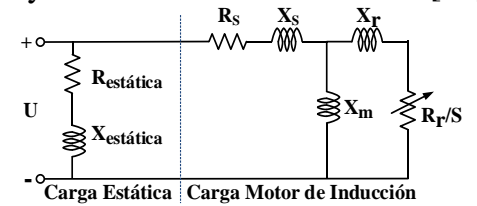

Figura 1: Circuito Equivalente Motor de Inducción

$$
\begin{aligned}
& \begin{aligned}
\frac{d E_{d}}{d t}=-\frac{1}{T_{o}^{\prime}}\left(\frac{x}{x^{\prime}} E_{d}-\right. & \left.\frac{x-x}{x^{\prime}} U_{d}\right) \\
& +2 \pi f_{o} s E_{q}
\end{aligned} \\
& \begin{aligned}
\frac{d E_{q}}{d t}=-\frac{1}{T_{o}^{\prime}}\left(\frac{x}{x^{\prime}} E_{q}-\frac{x-x}{x^{\prime}} U_{q}\right) \\
+2 \pi f_{o} s E_{d}
\end{aligned} \\
& \frac{d s}{d t}=-\frac{1}{H}\left[P_{m}-P_{m o}\left(A \omega^{2}+B \omega+C\right)\right]
\end{aligned}
$$

\section{ESTABILIDAD DINÁMICA DE TENSIÓN EN MICRORREDES}

Un sistema eléctrico se dice que es estable en términos de tensión, si después de cualquier perturbación es capaz de restaurar la tensión a un estado estacionario y mantener la magnitud de tensión estable. La principal condición de la inestabilidad de tensión es que el sistema eléctrico no puede satisfacer la demanda de potencia reactiva, esto puede ser causado por los límites en la corriente de excitación de los generadores, líneas de transmisión altamente inductivas, características de la carga o incluso los dispositivos de control de tensión en las redes de transmisión y distribución, que actúan como soporte de tensión. 
Otro fenómeno que a menudo es mencionado con la inestabilidad es el colapso de tensión. Éstos se tratan de dos diferentes conceptos. Inestabilidad de tensión es un fenómeno local, pero puede tener un impacto generalizado. El colapso de tensión es más complejo y es el resultado de una secuencia de eventos, que acompañan a la inestabilidad de tensión y que conduce a una caída de tensión significativa en la red eléctrica. La inestabilidad de tensión a veces se asocia con la inestabilidad de frecuencia. La pérdida gradual del sincronismo de generadores desencadenaría en una baja tensión en puntos intermedios de la red. Particularmente, en los sistemas de microrredes con integración de energías renovables, la alta demanda de potencia reactiva puede ser manipulada para llevar a escenarios de inestabilidad de tensión [23].

Con la presencia de ERNCs en MREs, el control de la generación se vuelve significativamente más desafiante en términos de ingeniería, debido a la mayor intermitencia e incertidumbre de los recursos energéticos primarios para mantener el equilibrio generacióndemanda. Con el objetivo de solucionar los inconvenientes técnicos que faciliten una adecuada operatividad de los sistemas de MREs y GD, en torno a flujos de potencia bidireccionales, perfiles de tensión dentro de los rangos establecidos, incertidumbre en la generación de electricidad, cargabilidad de los elementos del sistema eléctrico, y en general la utilización eficiente de los SSEE manteniendo la seguridad operativa, se demanda una mayor dependencia de sistemas y acciones de control [24].

No obstante, con las actuales redes de distribución evolucionando en MREs, las caídas de tensión y los límites de cargabilidad asociados a las corrientes circulantes, pueden convertirse en un problema de considerable representatividad, en particular con las redes de características eléctricas débiles y antiguas en función de su tiempo de operación [25]. Por lo que, el colapso de tensión, es decir, la disminución lenta y sostenida de la tensión asociada con el proceso de recuperación de carga y capacidad de suministro de potencia reactiva, se convierte en un desafío actual de análisis e investigación en las MREs. El funcionamiento operativo óptimo de las MREs, desde un punto de vista técnico y económico, guarda estrecha vinculación con las características topológicas de los elementos del sistema eléctrico, la cantidad y la ubicación de los componentes tipo GD. Este tipo de GD asociada a la conexión a un sistema tipo red de distribución débil, aumenta los niveles de cortocircuito, dando lugar a la aparición de fluctuaciones de tensión y, por ende, reducción de los márgenes de estabilidad del sistema MRE, por lo que es imperativo considerar nuevas técnicas y estrategias de control, que tomarán connotada influencia para contrarrestar esta problemática [26].

Los términos relacionados a estabilidad de tensión en MREs incluyen: calidad, regulación, distorsión y perfil de tensión. Como se indicó, la estabilidad de tensión en microrredes, consiste en mantener estable la amplitud de tensión en un nivel requerido por el sistema. Las principales causas que provocan problemas de estabilidad de tensión son: límites de potencia reactiva, cargas dinámicas y cambiadores de TAPs en transformadores, equipamiento de electrónica de potencia para el acoplamiento de GD en la MRE [27].

Con las tendencias actuales a que los sistemas eléctricos sean operados más cerca de sus límites, las estrategias operativas serán mucho menos predecibles. Por lo tanto, a medida que aumenta la tensión en una MRE estas no linealidades se tornan cada vez más pronunciadas. La no linealidad de la carga y las dinámicas asociadas a la GD son importantes a la hora de determinar inestabilidades. Por lo que, es fundamental considerar los diseños dinámicos de los controladores de los componentes de los sistemas de MREs [28].

Con respecto al control dinámico de la tensión, el estándar IEEE 1547-2018 proporciona los requisitos específicos con los que deben contar las unidades de GD, cuyo acoplamiento eléctrico a MREs es a través de inversores electrónicos. Por lo que, se permite el soporte dinámico de tensión en condiciones de falla o en transitorios de tensión, otorgando al inversor la capacidad de establecer ajustes al unísono o separadas para las potencias activa y reactiva [8].

\section{1 Índice de Desempeño de Tensión Dinámica}

Idealmente, el comportamiento de la tensión en función del tiempo cuando no existe la presencia de una perturbación en el sistema su valor es cercano a 1 p.u. Situación diferente ocurre cuando el sistema se encuentra sometido a alguna contingencia que genera una perturbación, por lo que, la dinámica transitoria de éste proceso ocasiona variaciones en la magnitud de tensión del sistema. A través de la Fig. 2 y la expresión (2), se muestra el análisis efectuado para determinar el índice de desempeño de la tensión dinámica (IDTD), que está basado en resultados discretizados de la magnitud de tensión a través de una simulación en el dominio del tiempo. A través del cálculo de éste índice, se puede determinar el desempeño y estabilidad de tensión. Cabe destacar que la formulación y la propuesta para el cálculo del IDTD es de elaboración propia de los autores.

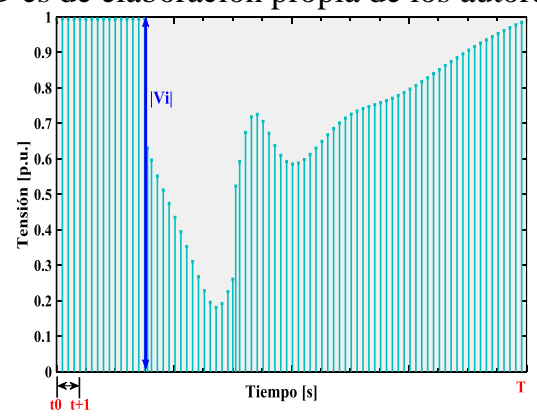

Figura 2: Comportamiento Dinámico de Tensiones Nodales sin DSTATCOM 


$$
I D T D=\sum_{t_{i}=0}^{T} \sum_{i=1}^{N}\left[t_{i} \times\left|V_{i}\right|\right] ; \forall t_{i} \in[0, T] ; \forall V_{i} \in[N]
$$

Donde: $\boldsymbol{V}_{\boldsymbol{i}}$ representa la magnitud de tensión de la señal de simulación para el tiempo $\boldsymbol{t}_{\boldsymbol{i}}$ y $\boldsymbol{N}$ representa la cantidad total de muestras de magnitud de tensiones discretizadas en la ventana de tiempo $\boldsymbol{T}$ de análisis. Es preciso indicar que mientras el IDTD es más cercano al producto de la tensión pre-falla por el tiempo $\boldsymbol{T}$, quiere decir que el IDTD tiene un mejor rendimiento en términos de respuesta de la tensión dinámica de la MRE. La eficacia del índice permite cuantificar las mejoras en la estabilidad dinámica de tensión (variabilidad de tensión en el tiempo) con la incorporación del dispositivo DSTATCOM operando coordinadamente con los componentes de GD en la MRE. Por ejemplo, para los casos de estudio de este artículo en condiciones estables, sin la presencia de perturbaciones en la microrred y para un tiempo de $5 \mathrm{~s}$, el IDTD sería igual a: $1 \times 5=5$.

\section{TECNOLOGÍA FACTS}

Según el IEEE, define a los FACTS como sigue: "Sistemas de transmisión de corriente alterna que incorporan controladores basados en electrónica de potencia y otros controladores estáticos, para mejorar la controlabilidad e incrementar la capacidad de transferencia de potencia" [18]. Desarrollos e investigaciones recientes han dado como resultado capacidades de control mejoradas en tiempo real, como la inyección y absorción de potencia reactiva de los FACTS, proporcionando así, oportunidades para el control y estabilidad de tensión y frecuencia en los sistemas eléctricos y particularmente en la actualidad en microrredes eléctricas [13], [19], [20].

De los diferentes dispositivos desarrollados en base a la tecnología FACTS, se puede mencionar el compensador estático sincrónico a nivel de distribución (DSTATCOM). En la Tabla 1, se expone resumidamente las características y atributos de control más destacados de este componente [21].

Tabla 1: Características y Atributos de Control DSTATCOM

\begin{tabular}{|c|c|c|}
\hline FACTS & Características & $\begin{array}{c}\begin{array}{c}\text { Atributos de } \\
\text { Control }\end{array} \\
\end{array}$ \\
\hline $\begin{array}{l}\text { Compensador } \\
\text { Estático } \\
\text { Sincrónico de } \\
\text { Distribución } \\
\text { DSTATCOM }\end{array}$ & $\begin{array}{l}\text { Es un generador sincrónico } \\
\text { estático, operando como un } \\
\text { compensador shunt de } \\
\text { potencia reactiva variable, } \\
\text { cuya corriente inyectada de } \\
\text { características capacitiva o } \\
\text { inductiva puede ser } \\
\text { controlada en forma } \\
\text { independiente de la tensión } \\
\text { del sistema eléctrico. }\end{array}$ & $\begin{array}{l}\text { Estabilidad y } \\
\text { Control Dinámico } \\
\text { de Tensión. } \\
\text { Control de Tensión } \\
\text { en Estado Estable. } \\
\text { Control y Gestión } \\
\text { de Potencia } \\
\text { Reactiva. } \\
\text { Amortiguamiento } \\
\text { de Oscilaciones. }\end{array}$ \\
\hline
\end{tabular}

\subsection{Compensador Estático Sincrónico para Distribución (DSTATCOM)}

El funcionamiento de un dispositivo DSTATCOM en conexión shunt en un PCC (Punto de Conexión Común
MRE-Sistema de Distribución), actúa en su operación como un compensador estático de $\mathrm{VAr}$, ya sea de características inductivas o capacitivas, independientemente de la tensión del sistema al que esté conectado y sin la necesidad de utilizar grandes bancos de capacitores o reactores. La característica principal de este equipo es proporcionar soporte de tensión al sistema. En la Fig. 3, se muestra el esquema y conexión de un DSTATCOM a través de un transformador acoplador [20]. La tensión de salida $\boldsymbol{V}_{\boldsymbol{s h}}$ que suministra el dispositivo será a frecuencia fundamental y de secuencia positiva. El DSTATCOM suministra una corriente $\boldsymbol{I}_{\boldsymbol{s h}}$ al sistema. Fasorialmente $\boldsymbol{V}_{\boldsymbol{j}}=\boldsymbol{V}_{\boldsymbol{s h}}+\boldsymbol{j} \boldsymbol{I}_{\boldsymbol{s h}} \boldsymbol{X}_{\boldsymbol{s} \boldsymbol{h}}$ donde $\boldsymbol{X}_{\boldsymbol{s h}}$ representa la reactancia del transformador acoplador. Por lo que, la tensión de salida del DSTATCOM es $\boldsymbol{V}_{\boldsymbol{s} \boldsymbol{h}}$ controlable tanto en su magnitud como en su fase.

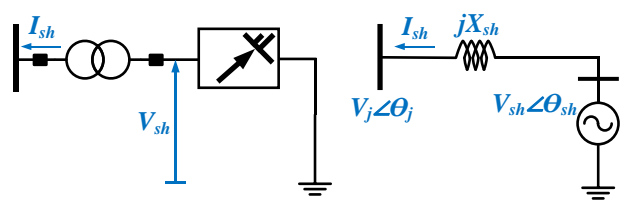

Figura 3: Esquema y diagrama de conexión DSTATCOM

\subsubsection{Modelo DSTATCOM Simplificado}

Para el modelado del dispositivo DSTATCOM, se ha considerado un generador estático a través de una fuente de corriente controlable [22]. El objetivo de este modelo simplificado es la compensación de potencia reactiva en la microrred, por lo tanto, la parametrización se realiza bajo este enfoque. Es así que, la fuente debe ser capaz de inyectar una corriente que esté en cuadratura con la tensión nodal donde se conecte el dispositivo. El esquema del controlador y de la fuente de corriente del modelo desarrollado se presentan en la Fig. 4.

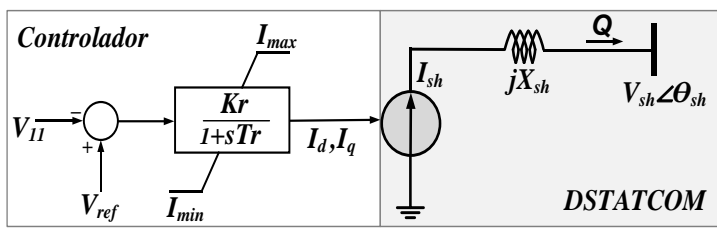

Figura 4: Controlador y Modelo DSTATCOM Simplificado

El controlador para este modelo, consiste en una función de transferencia de primer orden no lineal, que se emplea para conocer la respuesta dinámica del DSTATCOM. Donde $\boldsymbol{K}_{\boldsymbol{r}}$ es la ganancia del controlador, $\boldsymbol{T}_{\boldsymbol{r}}$ es la constante de tiempo de retardo del controlador, $\boldsymbol{V}_{\boldsymbol{r} \boldsymbol{e f}}$ es la tensión nodal de referencia de la MRE, $\boldsymbol{V}_{\mathbf{1 1}}$ es la tensión nodal a controlar en donde está conectado el DSTATCOM y $\boldsymbol{I}_{\boldsymbol{s} \boldsymbol{h}}$ es la corriente de compensación inyectada (la cual estará en función de sus componentes de ejes directo y cuadratura), con sus respectivos limites inferior y superior expresados en p.u. Las señales de corriente del controlador $\boldsymbol{I}_{\boldsymbol{s} \boldsymbol{h}}$ son expresadas a través de su descomposición en $\boldsymbol{I}_{\boldsymbol{d}}$ e $\boldsymbol{I}_{\boldsymbol{q}} ; \boldsymbol{V}_{\boldsymbol{r}} \boldsymbol{y} \boldsymbol{V}_{\boldsymbol{i}}$ son los módulos de la tensión en su ejes real e imaginario, respectivamente. A través de (3) se muestran las expresiones que gobiernan el desempeño de la fuente de corriente controlable. 


$$
I_{s h}=\left[i_{d} \frac{V_{r}}{V}-i_{q} \frac{V_{i}}{V}\right]+j\left[i_{d} \frac{V_{i}}{V}+i_{q} \frac{V_{r}}{V}\right]
$$

La potencia inyectada por la fuente controlable se expresa a través de (4), donde se debe considerar que el requerimiento de modelado es únicamente para aporte de potencia reactiva, por lo tanto, la potencia activa es cero.

$$
\boldsymbol{P}+\boldsymbol{j} \boldsymbol{Q}=\left[\boldsymbol{V}_{\boldsymbol{r}} \boldsymbol{I}_{\boldsymbol{r}}+\boldsymbol{V}_{\boldsymbol{i}} \boldsymbol{I}_{\boldsymbol{i}}\right]+\boldsymbol{j}\left[\boldsymbol{V}_{\boldsymbol{i}} \boldsymbol{I}_{\boldsymbol{r}}-\boldsymbol{V}_{\boldsymbol{r}} \boldsymbol{I}_{\boldsymbol{i}}\right]=\mathbf{0}+\boldsymbol{j} \boldsymbol{Q}
$$

En consecuencia, al desarrollar matemáticamente las ecuaciones (3) y (4), la corriente de inyección del dispositivo DSTATCOM, para el modelo simplificado, obedece el comportamiento operativo en concordancia a la expresión (5). Seguidamente, en la ecuación (6) se determina la expresión, mediante la cual el controlador permite que la corriente de inyección del dispositivo opere únicamente en cuadratura.

$$
\begin{gathered}
I_{s h}=\frac{I_{r}}{V_{i}} V \\
I_{s h}=-I_{q}
\end{gathered}
$$

Por consiguiente, en la Tabla 2, se presentan los parámetros y valores del controlador desarrollado para éste modelo de DSTATCOM.

Tabla 2: Parámetros Controlador DSTATCOM Simplificado

\begin{tabular}{|c|c|c|c|}
\hline Parámetro & Valor & Parámetro & Valor \\
\hline $\mathrm{Kr}$ & 100 & I_min & -1 \\
\hline $\mathrm{Tr}$ & 0,05 & I_max & 1 \\
\hline
\end{tabular}

\subsubsection{Modelo DSTATCOM basado en control PWM}

El modelo de DSTATCOM desarrollado en esta sección, corresponde a un conversor electrónico de dos niveles basado en PWM (Pulse-Width Modulation, modulación por ancho de pulsos) controlado. La potencia reactiva de entrega o recepción al sistema, que se desarrolla internamente en un dispositivo DSTATCOM, se genera a través de una onda de tensión sincrónica en sus termínales utilizando convertidores GTO. En la Fig. 5 , se muestra esquemáticamente el dispositivo DSTATCOM con su controlador basado en PWM.

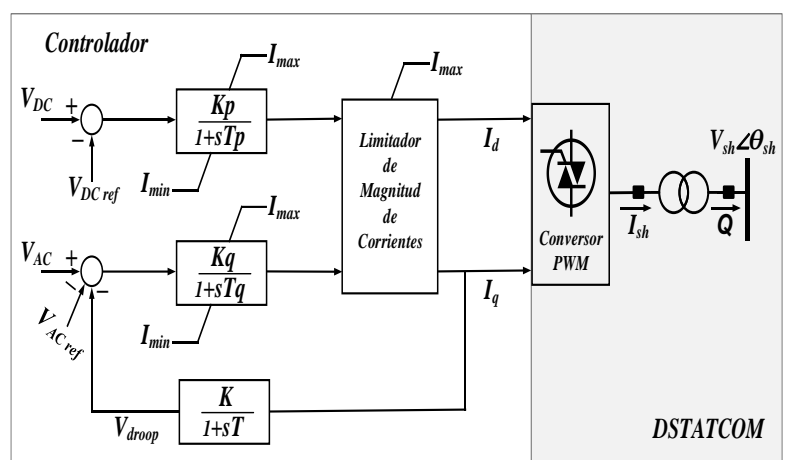

Figura 5: Controlador y Modelo DSTATCOM basado en PWM

El esquema del controlador consiste en establecer acciones de control tanto para las componentes de corrientes contínua como alterna del conversor. En ésta última corriente se establece un lazo de retroalimentación con control de caída (droop), que permita establecer un comparativo de error en la señal de tensión con respecto a la tensión de referencia de la MRE. Las señales de corrientes AC y DC están limitadas en los bloques integradores. Finalmente, el bloque limitador de corrientes permite controlar las señales en eje directo y cuadratura al conversor PWM. En la Tabla 3, se presentan los parámetros y valores del controlador, para este modelo de dispositivo DSTATCOM.

Tabla 3: Parámetros Controlador DSTATCOM control PWM

\begin{tabular}{|c|c|c|c|}
\hline Parámetro & Valor & Parámetro & Valor \\
\hline $\mathrm{Kp}$ & 10 & $\mathrm{~K}$ & 0,05 \\
\hline $\mathrm{Tp}$ & 0,002 & $\mathrm{~T}$ & 0,001 \\
\hline $\mathrm{Kq}$ & 4 & I_min & -2.67 \\
\hline $\mathrm{Tq}$ & 0,002 & I_max & 2,67 \\
\hline droop & 0,005 & & \\
\hline
\end{tabular}

\section{CASOS DE ESTUDIO, SIMULACIONES Y RESULTADOS COMPARATIVOS}

Los modelos presentados en las secciones anteriores y el sistema de MRE, fueron implementados en el paquete computacional PowerFactory DIgSILENT, que es utilizado como herramienta para realizar las diferentes simulaciones en el dominio del tiempo en una ventana temporal de $5 \mathrm{~s}$, que guarda concomitancia con los estudios de estabilidad de tensión de corto plazo. Los detalles de los modelos desarrollados y los resultados obtenidos para el análisis de estabilidad dinámica de tensión en una microrred eléctrica son presentados y discutidos en esta sección.

El sistema de microrred de prueba de la CIGRÉ [24] es utilizado para el análisis y validación de los modelos de DSTATCOM desarrollados. En la Fig. 6, se presenta el sistema de MRE empleado, considerando dos tipos de tecnologías de GD: Sistema Solar Fotovoltaico y Generación Sincrónica térmica (equipado con AVR y gobernador: ESDC1A y TGOV1, respectivamente) [24]. Además, se presentan cargas estáticas (con una participación aproximada del $30 \%$ de la carga total de la MRE) y de tipo motor de inducción (con $70 \%$ de participación), las mismas que se encuentran conectadas en los diferentes nodos de la MRE. Un total de 105 MIs se han incorporado al sistema de MRE, los cuales han sido distribuidos uniformemente a razón de 15 unidades de MI en cada uno de los nodos: 2, 3, 4, 7, 8, 11 y 12 que conforman la MRE. Particularmente, en lo que corresponde a la carga dinámica de tipo de motor de inducción, sus parámetros característicos se han modelado en concordancia a los siguientes datos: $\mathrm{P}=250$ $\mathrm{kW}$; Momento de Inercia $=21,624 \mathrm{kgm}^{2} ; \mathrm{R}_{\mathrm{s}}=0,035821$ p.u.; $X_{\mathrm{s}}=0,01$ p.u.; $\mathrm{X}_{\mathrm{m}}=1,55462$ p.u.; $\mathrm{R}_{\mathrm{r}}=0,010807$ p.u.; $\mathrm{X}_{\mathrm{r}}=0,421021$ p.u.; $\mathrm{S}=0.224$ [17].

Para los dos modelos de DSTATCOM, simplificado y basado en PWM se ha considerado una potencia nominal de $5000 \mathrm{kVAr}$. Cabe destacar que los esquemas de control asociados a ambos dispositivos están 
diseñados con el objetivo de operar únicamente en situaciones bajo la presencia de perturbaciones o contingencias en la MRE. La condición operativa pre contingencia del sistema MRE se resume en la Tabla 4.

Tabla 4: Condiciones Operativas del Sistema de Microrred
\begin{tabular}{|c|r|r|}
\hline Tipo de Generación & P [kW] & Q [kVAr] \\
\hline Sincrónica Diésel & 32538,34 & 26355,37 \\
\hline Solar Fotovoltaica & 5000,00 & 0,00 \\
\hline Importación Red & 35,79 & 181,83 \\
\hline Tipo de Carga & $\mathbf{P}[\mathbf{k W}]$ & $\mathbf{Q}[\mathbf{k V A r}]$ \\
\hline Cargas Dinámicas MI & 26196,08 & 19647,01 \\
\hline Cargas Estáticas P-Q & 10463,00 & 4682,43 \\
\hline Pérdidas & 915,05 & 2207,76 \\
\hline
\end{tabular}

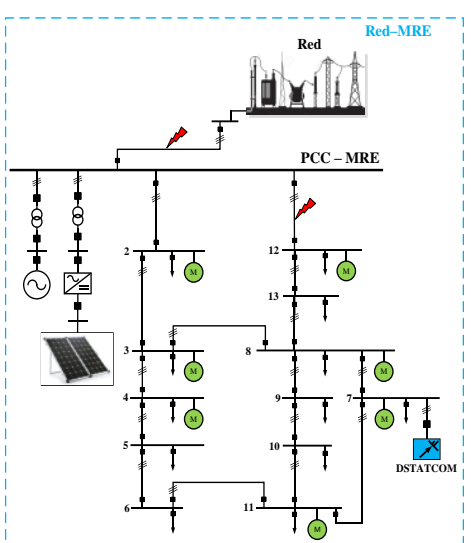

Figura 6: Modelo de Microrred de Prueba de la CIGRÉ [24]

Las contingencias a tomar en consideración corresponden a un criterio $\mathrm{N}-2$, este tipo de perturbaciones son consideraras de poca probabilidad de ocurrencia, pero que cuando ocurren comprometen severamente la operatividad del sistema de microrred, este tipo de contingencias son conocidos como eventos resilientes [1], [21]. La primera contingencia se considera a la desconexión del enlace de interconexión red-MRE, por consiguiente, la segunda contingencia se considera la desconexión ocasionada por una falla en el enlace PCC12 de la MRE. Para ambas contingencias se considera una falla trifásica al $50 \%$ de la longitud de los alimentadores, coincidente en el tiempo a los $0,15 \mathrm{~s}$ de iniciado el proceso de simulación, además, se considera un tiempo de $0,15 \mathrm{~s}$ para el proceso de detección y despeje de ambas fallas. No se consideró fallas o salidas de los componentes de GD justamente porque se quiere evaluar la capacidad de éstos y el accionar de los sistemas de control desarrollados, específicamente el sistema solar fotovoltaico en relación al soporte dinámico de tensión y suministro de potencia reactiva en presencia de contingencias acorde a lo estipulado en el estándar IEEE1547-2018.

Los nodos considerados para el análisis son: PCC, 4, 7 y 11, que corresponden a la MRE de la Fig. 6. La elección de los nodos de análisis se justifica debido a que en el nodo PCC se encuentra conectado el parque generador de la MRE. El nodo 11 se lo consideró por ser el nodo más lejano eléctricamente desde el PCC. En relación a los nodos 4 y 7 se consideraron por ser estos nodos los que se encuentran en el baricentro topológico de la microrred, todos los nodos de análisis tienen presencia de cargas de tipo MI. A través de la Fig. 7, se muestra el comportamiento dinámico de las tensiones nodales en la MRE ante la presencia de las contingencias descritas en anterioridad ( $\mathrm{N}-2)$ y sin la conexión de ningún dispositivo de compensación tipo DSTATCOM.

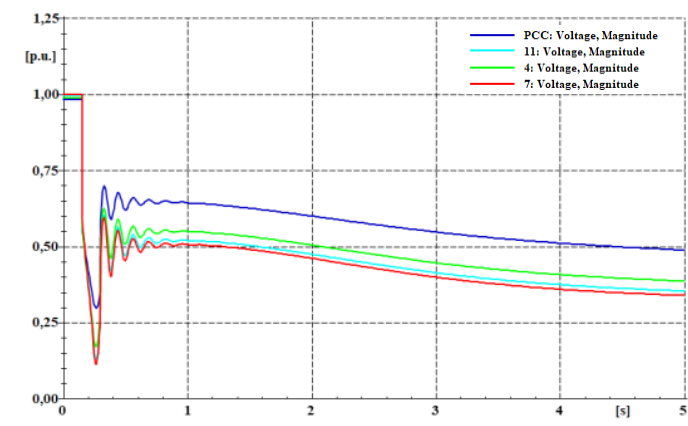

Figura 7: Comportamiento Dinámico de Tensiones Nodales sin DSTATCOM

De los resultados obtenidos por simulación para este caso de estudio, es posible concluir que se produce un colapso asociado a un proceso de inestabilidad de tensión, esto debido a que no se visualiza recuperación de las tensiones dinámicas nodales posterior a la ocurrencia de las contingencias.

A través de una técnica de búsqueda exhaustiva [29] y, considerando los resultados obtenidos de las simulaciones dinámicas, se determinará la mejor ubicación para la conexión del DSTATCOM. En la sección siguiente se detalla con mayor rigurosidad el procedimiento a realizar.

\subsection{Ubicación óptima del dispositivo DSTATCOM en función de su respuesta dinámica de tensión.}

El comportamiento de estabilidad dinámica de tensión en la MRE se evalúa para cada modelo de DSTATCOM. Además, a través del IDTD se determina el mejor desempeño de entre los dos modelos desarrollados y su ubicación óptima para la conexión de este dispositivo en la MRE. La conexión de los modelos DSTATCOM fueron conectados alternadamente en los nodos: PCC, 4, 7 y 11. En el procedimiento de búsqueda óptima para la ubicación y conexión del dispositivo DSTATCOM, se consideró el algoritmo de búsqueda exhaustiva, cuyo desempeño y evaluación se basa en las simulaciones y resultados del IDTD en concordancia al diagrama de flujo mostrado en la Fig. 8. 


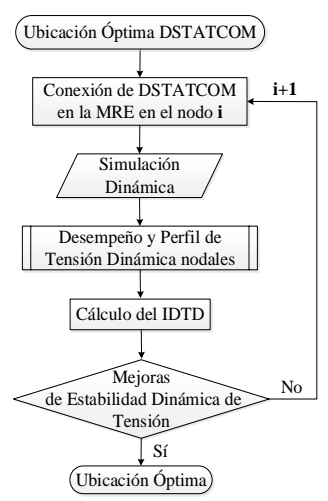

Figura 8: Algoritmo de Búsqueda Exhaustiva para DSTATCOM

Los resultados obtenidos, que identifican el comportamiento de las tensiones nodales en la MRE para cada modelo de DSTATCOM teniendo en cuenta las simulaciones empleadas en función del algoritmo de búsqueda empleado, son mostrados en la Fig. 9.

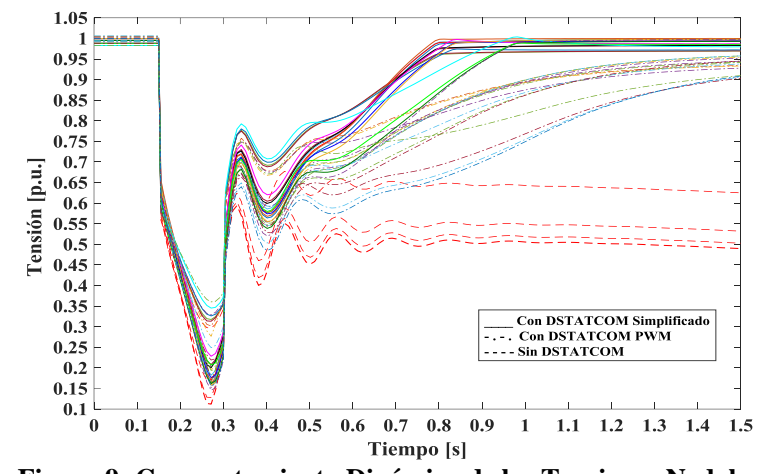

Figura 9: Comportamiento Dinámico de las Tensiones Nodales para diferentes posiciones de los modelos DSTATCOM

El criterio de evaluación para determinar la mejor ubicación del DSTATCOM y con ello mejorar el desempeño y estabilidad de la tensión dinámica en la MRE, fue realizado con base en el cálculo del IDTD expuesto en la sección 4.1, donde para cada ubicación del dispositivo DSTATCOM se procedió con su cálculo. En la Tabla 5 se presentan los resultados obtenidos para el IDTD y se muestra comparativamente, que la ubicación optima de conexión de ambos modelos de DSTATCOM desarrollados, corresponden a la conexión en nodo 7 .

\begin{tabular}{|c|c|c|c|c|c|c|c|c|}
\hline \multirow{2}{*}{ UD } & \multicolumn{2}{|c|}{ PCC } & \multicolumn{2}{|c|}{4} & \multicolumn{2}{|c|}{7} & \multicolumn{2}{|c|}{11} \\
\hline & $\mathbf{S}$ & P & $\mathbf{S}$ & $\mathbf{P}$ & $\mathbf{S}$ & $\mathbf{P}$ & $\mathbf{S}$ & $\mathbf{P}$ \\
\hline PCC & 4,73 & 4,45 & 4,69 & 4,37 & 4,71 & 4,38 & 4,70 & 4,37 \\
\hline 4 & 4,70 & 4,50 & 4,74 & 4,51 & 4,76 & 4,52 & 4,75 & 4,51 \\
\hline 7 & 4,69 & 4,52 & 4,72 & 4,53 & 4,78 & 4,58 & 4,76 & 4,56 \\
\hline 11 & 4,69 & 4,53 & 4,72 & 4,54 & 4,77 & 4,58 & 4,76 & 4,58 \\
\hline
\end{tabular}

UD: Ubicación DSTATCOM; N: Nodo de MRE; S: DSTATCOM modelo Simplificado; P: DSTATCOM modelo basado en PWM

Con el dispositivo DSTATCOM conectado en el nodo 7 de la MRE, a continuación, en las Figs. 10 y 11, se muestran los desempeños de las tensiones nodales de la MRE para los modelos de DSTATCOM simplificado y PWM controlado, respectivamente. Visiblemente se observan mejoras en el desempeño dinámico de las tensiones nodales para ambos casos. Con lo cual, se ha demostrado concluyentemente las mejoras obtenidas en la estabilidad dinámica de tensión para la operación asilada de la MRE en comparativa con el escenario en donde no estuvo conectado ningún equipamiento de compensación sincrónica estática.

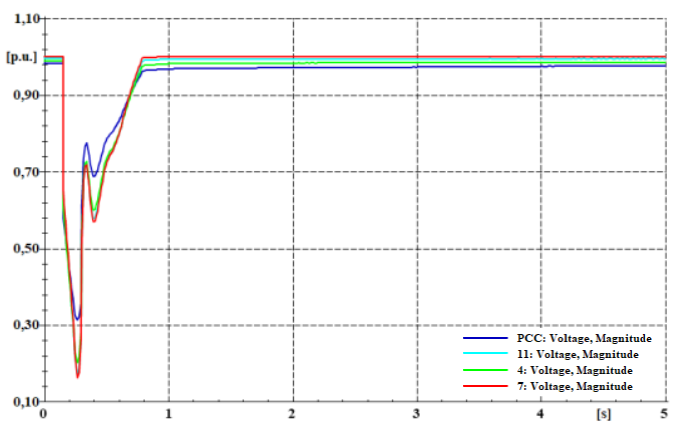

Figura 10: Dinámica de tensión modelo DSTATCOM simplificado

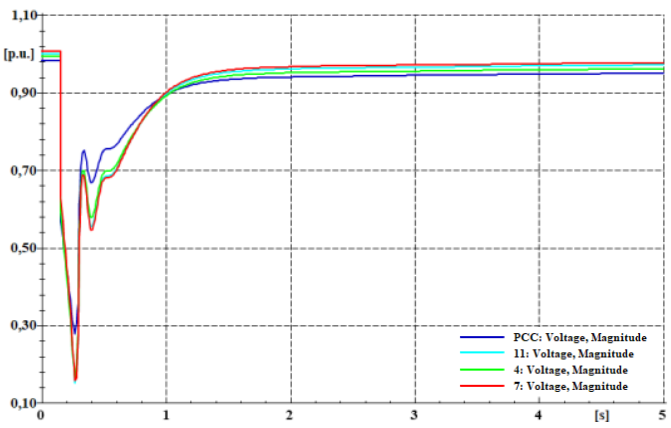

Figura 11: Dinámica de tensión modelo DSTATCOM PWM

En función de los resultados obtenidos para el IDTD a través de la Fig. 12, se muestra comparativamente el desempeño de cada modelo DSTATCOM desarrollados.

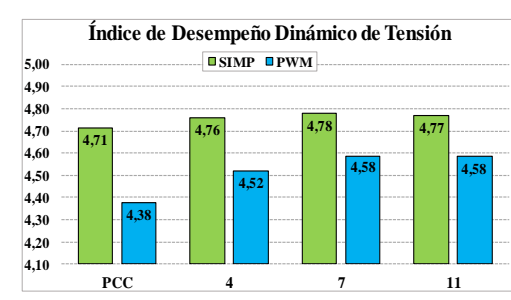

Figura 12: IDTD comparativo entre Modelos DSTATCOM usados

Por lo tanto, se puede concluir que en términos de desempeño y estabilidad de tensión dinámica en la MRE el DSTATCOM en su modelo simplificado otorga una mejora un tanto superior que su par basado en PWM. Por otro lado, el DSTATCOM en su modelo de control a base de PWM, otorga también mejoras en la estabilidad dinámica de tensión y muestra un suavizado de oscilaciones posterior a la ocurrencia de las perturbaciones, tal cual se mostró en la Fig. 10 de la MRE. Comparativamente y considerando términos porcentuales promediados acorde a la Fig. 12, el modelo de DSTATCOM basado en control PWM tiene un desempeño menor en el orden del 5\% que el modelo de DSTATCOM simplificado, lo cual no implica una 
variabilidad considerable entre los modelos debido a que es concordante con el criterio de diseño y modelos en ingeniería. La variabilidad de éste modelo obedece justamente al detalle de componentes empleados en el modelamiento, lo que desencadena en pequeños retardos al momento del cálculo de simulación dinámica.

A través de la Fig. 13, se muestran comparativamente los desempeños asociados a las tensiones dinámicas nodales de la MRE. Como se expuso en anterioridad, acá se presentan los resultados obtenidos para los tres casos de la microrred: sin equipo de compensación, DSTATCOM modelo simplificado y DSTATCOM en su modelo de control a base de PWM.

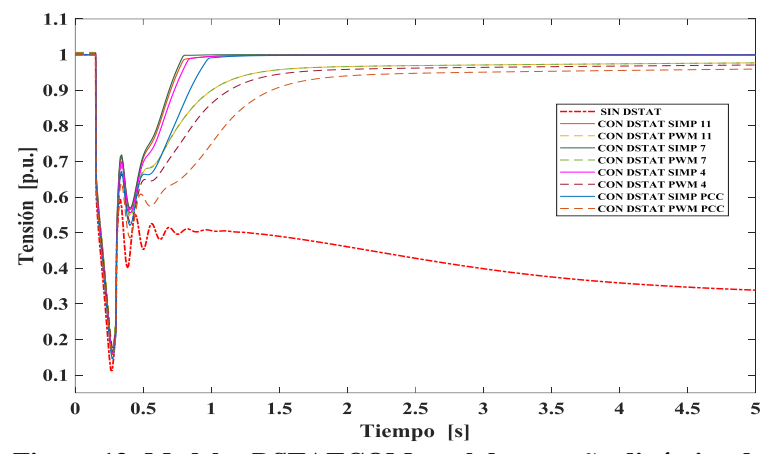

Figura 13: Modelos DSTATCOM en el desempeño dinámico de tensiones nodales en la MRE

Para ambos modelos desarrollados de DSTATCOM se observa que ante la ocurrencia de las contingencias que desencadenaron la operación aislada de la MRE, ésta ha respondido operativamente de manera correcta, la tensión se ha restablecido favorablemente, con ello se puede garantizar que las cargas dinámicas sensibles de tipo motóricas puedan seguir funcionando sin riesgo a incurrir en inconvenientes abruptos de desaceleración que restrinja el funcionamiento de este tipo de cargas.

En concomitancia con el objetivo de la operación de los dispositivos DSTATCOM, en la Fig. 14, se muestran los comportamientos en términos de potencia reactiva, como era de esperarse, el aporte y respuesta de ésta potencia se muestra en coherencia con la ocurrencia de las perturbaciones, es decir, la mayor contribución de potencia reactiva se suministra durante los cortocircuitos, esto ha permitido mantener y realizar soporte dinámico de tensión y el amortiguamiento de oscilaciones.

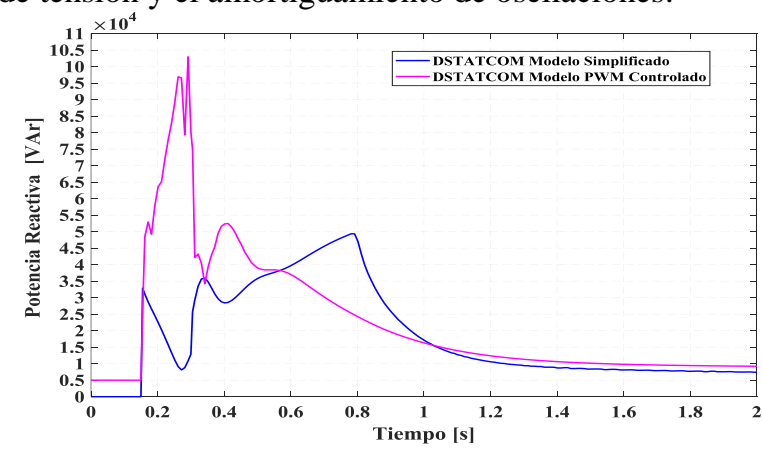

Figura 14: Respuesta de Potencia Reactiva DSTATCOM modelo Simplificado y PWM controlado
La variabilidad de respuesta entre los dos modelos presentados, radica en la complejidad del modelado empleado, para el modelo simplificado se ve una respuesta mejor en amortiguamiento de oscilaciones y recuperación de la magnitud de tensión cercana a 1 p.u. (ver Fig. 13). A su vez, para el modelo de basado en PWM controlado, también se aporta mitigando las oscilaciones de tensión, permitiendo un suavizado retardado, esto obedece a la característica de control empleada básicamente en la etapa del PWM y en los tiempos de conmutación de los controles realizados.

Para ambos dispositivos DSTATCOM, la respuesta al momento de iniciada las perturbaciones en el sistema de MRE presenta buenas características de desempeño, esto es, realizando las compensaciones de potencia reactiva satisfactoriamente. Posterior al despeje de las fallas y en el modo operativo en isla de la MRE, se aprecia que la tensión presenta un comportamiento estable para ambos escenarios, por su parte, el aporte de potencia reactiva transitorio se ve disminuido considerablemente a partir de $\operatorname{los} 1 \mathrm{~s}$, como se aprecia en la Fig. 14. Esto obedece a la respuesta coordinada de operación entre la generación sincrónica y solar fotovoltaica, quienes ante el asilamiento permiten mantener operativa la MRE. Por lo que, los modelos empleados en esta investigación han respondido correctamente a su dinámica operacional y en concordancia con las características operativas propias de un dispositivo DSTATCOM y de control coordinado entre los componentes de GD y cargas que conforman la microrred.

\section{CONCLUSIONES}

Si bien es cierto, que los dispositivos de tecnología FACTS han alcanzado cierta madurez y posicionamiento en los grandes sistemas de potencia, con el actual auge de los sistemas de GD y MREs, en la literatura se ha encontrado pocos aportes a la implementación de éstos dispositivos para el estudio y análisis en dichos sistemas. A través de este artículo se ha mostrado la importancia de implementar un dispositivo DSTATCOM en un sistema de MRE, cuyo componente de carga corresponde a una alta penetración de motores de inducción, quienes comprometen trascendentalmente la estabilidad y control dinámico de la tensión. Los casos de estudio se consideraron bajo la presencia de un evento extremo resiliente asociado a la ocurrencia simultánea de una doble contingencia, que desencadena en la operación aislada de la MRE. Con esto, se ha determinado las acciones de control necesarias para evitar inestabilidades de tensión, por lo que, se desarrollaron dos tipos de modelos de DSTATCOM, para mejorar el desempeño dinámico en términos de estabilidad de tensión en una MRE. Bajo este escenario se estableció la ubicación óptima para la conexión del DSTATCOM en la MRE a través de un algoritmo de búsqueda exhaustiva y con base en los resultados del Índice de Desempeño de Tensión Dinámica propuesto por los autores. 
Los resultados obtenidos a través de simulaciones y del IDTD, comparativamente mostraron una adecuada operatividad de la MRE aislada con la presencia de los modelos DSTATCOM simplificado y PWM controlado, ambos aportando a mejorar la estabilidad dinámica para las tensiones nodales.

Como trabajos futuros se establece seguir investigando para establecer la calibración óptima de los parámetros asociados a los controladores DSTATCOM, atendiendo además un enfoque de resiliencia operativa basado en métricas y según los requerimientos establecidos en el estándar IEEE-1547-2018 para la operatividad estocástica de recursos primarios en MREs.

\section{REFERENCIAS BIBLIOGRÁFICAS}

[1] N. Mahdavi, S. Najafi, and N. Bizon, Power Systems Resilience. 2019.

[2] L. A. Paredes, B. R. Serrano, and M. G. Molina, "Microgrids - a Methodological Review in the Current Context of Electric Systems," CIEEPI - Rev. del Col. Ing. Eléctricos y Electrónicos Pichincha, no. 49, 2019.

[3] K. P. Schneider, F. K. Tuffner, M. A. Elizondo, C. C. Liu, Y. $\mathrm{Xu}$, and D. Ton, "Evaluating the Feasibility to Use Microgrids as a Resiliency Resource," IEEE Trans. Smart Grid, vol. 8, no. 2, pp. 687-696, 2017.

[4] D. E. Olivares et al., "Trends in microgrid control," IEEE Trans. Smart Grid, vol. 5, no. 4, pp. 19051919, 2014.

[5] J. Guerrero et al., "Distributed generation: Toward a new energy paradigm," IEEE Ind. Electron. Mag., vol. 4, no. 1, pp. 52-64, 2010.

[6] L. A. Paredes, B. R. Serrano, and M. G. Molina, "Energy Management in Isolated Microgrids of the Ecuadorian Amazon Region," in 3er Simposio Ibero-Americano en Microrredes Inteligentes con Integración de Energías Renovables, 2019.

[7] F. Katiraei, M. R. Iravani, and P. W. Lehn, "Microgrid autonomous operation during and subsequent to islanding process," IEEE Trans. Power Deliv., vol. 20, no. 1, pp. 248-257, 2005.

[8] IEEE PES Industry Technical Support Task Force, "Impact of IEEE 1547 Standard on Smart Inverters," 2018.

[9] A. Khadem Abbasi, M. W. B. Muatafa, and A. S. B. Mokhtar, "Small signal stability analysis of rectifierinverter fed induction motor drive for microgrid applications," IEEE Reg. 10 Annu. Int. Conf. Proceedings/TENCON, pp. 1015-1019, 2011.

[10]D. Wu, H. Wu, and H. Dongt, "Influence of induction motor starting on microgrid," Asia-Pacific Power Energy Eng. Conf. APPEEC, vol. 2018Octob, pp. 376-381, 2018.

[11] N. Afrin, F. Yang, J. Lu, and M. Islam, "Impact of induction motor load on the dynamic voltage stability of microgrid," ANZCC 2018 - 2018 Aust. New Zeal. Control Conf., pp. 397-402, 2018.

[12] W. Freitas, A. Morelato, W. Xu, and F. Sato, "Impacts of AC generators and DSTATCOM devices on the dynamic performance of distribution systems," IEEE Trans. Power Deliv., vol. 20, no. 2 II, pp. 1493-1501, 2005.

[13] T. Samad and A. M. Annaswamy, "Controls for Smart Grids: Architectures and Applications," Proc. IEEE, vol. 105, no. 11, pp. 2244-2261, 2017.

[14] L. A. Paredes, B. R. Serrano, and M. G. Molina, "Nuevo Paradigma de los Sistemas Eléctricos: Generación Distribuida y Microrredes Eléctricas un Vínculo de Accesibilidad a la Electricidad en América Latina y el Caribe," ENERLAC, vol. 3, 2020.

[15] M. S. Mahmoud and F. M. AL-Sunni, Control and Optimization of Distributed Generation Systems. 2015.

[16] G. B. Gharehpetian and S. M. M. Agah, Distributed Generation Systems: Design, Opearation and Grid Integration. 2017.

[17] E. A. Tapia, J. D. Pinzón, and D. G. Colomé, "Load Dynamic Impact on Short-Term Voltage Stability," in 2019 FISE-IEEE/CIGRE Conference - Living the energy Transition (FISE/CIGRE), 2019.

[18] A. A. Edris et al., "Proposed terms and definitions for Flexible AC Transmission System (FACTS)," IEEE Trans. Power Deliv., vol. 12, no. 4, pp. 18481853, 1997.

[19] L. Gyugyi, R. A. Otto, and T. H. Putman, "Principles and applications of static, thyristor-controlled shunt compensators," IEEE Trans. Power Appar. Syst., vol. PAS-97, no. 5, pp. 1935-1945, 1978.

[20] N. G. Hingoranl, L. Gyugyi, and M. E. El-Hawary, Understanding FACTS: Concepts and technology of flexible ac transmission systems. 1999.

[21]L. A. Paredes, B. R. Serrano, and M. G. Molina, "FACTS Technology to Improve the Operation of Resilient Microgrids,” in 2019 FISE-IEEE/CIGRE Conference - Living the Energy Transition (FISE/CIGRE), 2019, pp. 1-7.

[22]F. M. Gonzalez-Longatt and J. L. Rueda, PowerFactory Aplications for Power System Analysis. Springer, 2014.

[23] S. Houbing, S. Ravi, S. Tmim, and J. Sabina, Smart Cities, no. 10. Wiley, 2017. 
[24]L. A. Paredes, B. R. Serrano, and M. G. Molina, "Voltage Stability Improvement with a DSTATCOM in a Microgrid Integrated by DG Solar Photovoltaic and Conventional," Rev. Técnica “energía,” vol. 16, no. II, 2020.

[25] R. B. Prada, "Voltage stability and thermal limit: Constraints on the maximum loading of electrical energy distribution feeders," IEE Proc. - Gener. Transm. Distrib., vol. 145, no. ii, pp. 573-577, 1998.

[26] P. Coelho, M. Gomes, and C. Moreira, Microgrids Design and Implementation. 2019.

[27] Y. Xu, C.-C. Liu, K. Schneider, F. Tuffner, and D. Ton, "Microgrids for Service Restoration to Critical Load in a Resilient Distribution System," IEEE Trans. Smart Grid, vol. 9, no. 1, pp. 1-1, 2016.

[28] J. Hossain and H. R. Pota, Robust Control for Grid Voltage Stability: High Penetration of Renewable Energy. 2014.

[29]E. Rodríguez Tello, “Algoritmos de búsqueda exhaustiva," CINVESSTAV - Tamaulipas. 2018.

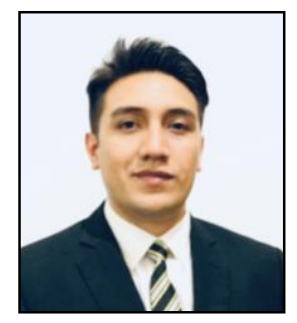

Luis A. Paredes. - Nació en Quito, Ecuador en 1987. Recibió su título de Ingeniero Eléctrico de la Escuela Politécnica Nacional en 2012 y de Magíster en Gestión de Energías en 2016. Además, ha realizado varios cursos y especializaciones en temáticas de energía eléctrica en Estados Unidos, China, Perú, Chile y Brasil. Su experiencia profesional ha sido desarrollada en varias empresas e instituciones del sector eléctrico y energético del Ecuador. Actualmente es candidato a Doctor en Ingeniería Eléctrica (Ph.D.) del Instituto de Energía Eléctrica (IEE) de la Universidad Nacional de San Juan (UNSJ) en Argentina. Sus campos de investigación están relacionados con: Resiliencia de los Sistemas Eléctricos, Estabilidad y Control en Microrredes, FACTS, Movilidad Eléctrica, Energías Renovables y Eficiencia Energética.

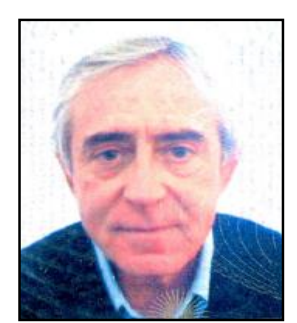

Benjamín R. Serrano. - Nació en San Juan, Argentina en 1955. Recibió su título de Ingeniero Electromecánico en la Universidad Nacional de San Juan (UNSJ), Argentina en 1981. Obtuvo su título de Doctor en Ingeniería Eléctrica en el Instituto de Energía Eléctrica (IEE) de la UNSJ, Argentina en 2017. Desde 2020 se desempeña como Vicedirector del Instituto de Energía Eléctrica (IEE) de la UNSJ-CONICET.
Actualmente desarrolla sus tareas docentes, de investigación y transferencia en temáticas relacionadas con la Programación Óptima de la Operación de los Sistemas Eléctricos de Potencia, considerando el Control de Tensiones y Suministro de Potencia Reactiva.

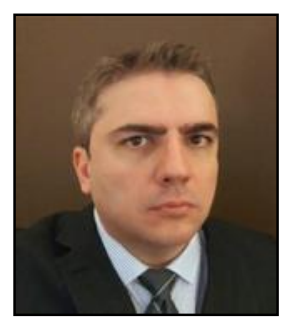

Marcelo G. Molina. - Es profesor titular de Electrónica de Potencia, Energías Renovables y Redes Eléctricas Inteligentes en la Universidad Nacional de San Juan (UNSJ), Argentina. Desde 2019 se desempeña como Director del Instituto de Energía Eléctrica (IEE) de la UNSJ-CONICET. El Dr. Molina recibió el título de Ingeniero Electrónico de la Universidad Nacional de San Juan, Argentina en 1997 y su Ph.D. de la misma universidad en 2004. Sus actividades de investigación se centran en el modelado, análisis y control de sistemas eléctricos de potencia, electrónica de potencia, tecnologías de microrredes, redes inteligentes y la aplicación de almacenamiento de energía. 